Artículo

\title{
Climas de Jalisco según el sistema Köppen-García con ajuste por vegetación potencial
}

\author{
José Ariel Ruiz-Corral ${ }^{1 \S}$ \\ Sergio Honorio Contreras Rodriguez ${ }^{1}$ \\ Giovanni Emmanuel García Romero ${ }^{2}$ \\ Raymundo Villavicencio García ${ }^{1}$
}

${ }^{1}$ Centro Universitario de Ciencias Biológicas y Agropecuarias-Universidad de Guadalajara. Carretera Guadalajara-Nogales km 15.5, Jalisco, México. (sergio.contreras@academicos.udg.mx; raymundo.villavicencio@academicos.udg.mx) ${ }^{2}$ Dirección de Medio Ambiente del municipio de Guadalajara. Av. Miguel Hidalgo y Costilla 426, Zona Centro, Guadalajara, Jalisco, México. CP. 44100. (geog.vanni@gmail.com).

${ }^{\S}$ Autor para correspondencia: ariel.ruiz@academicos.udg.mx.

\section{Resumen}

El objetivo de la presente investigación fue actualizar el mapa de climas del estado de Jalisco de acuerdo con la clasificación climática Köppen-García, con base en imágenes raster climáticas de alta resolución $(90 \mathrm{~m})$ y un ajuste por correspondencia climas-vegetación potencial. Se trabajó con datos climáticos diarios, mensuales y anuales de 154 estaciones meteorológicas del estado de Jalisco y 151 estaciones de estados vecinos. Los datos corresponden a la serie 1961-2014 en el caso de las estaciones de Jalisco y la serie 1961-2010 para estados vecinos. Con esta información se obtuvieron imágenes raster para la clasificación del clima con el método Köppen-García, mediante procesos de interpolación implementados con el método Anusplin. En el sistema Idrisi y utilizando rutinas de álgebra de mapas, se obtuvo el mapa de climas de Jalisco a nivel de tipo y subtipo climático. Dicho mapa se corrigió atendiendo a la correspondencia de los climas obtenidos con la vegetación potencial según COTECOCA. Los resultados mostraron correspondencia de $84 \%$ entre climas y tipos de vegetación y sólo $16 \%$ de los polígonos climáticos fueron corregidos. Se obtuvieron 29 variantes climáticas, de las cuales, los climas secos o semiáridos representan 13.78\% del territorio estatal, cálido húmedos $\mathrm{Aw}$ el 21.77\%, semicálidos húmedos $\mathrm{A}(\mathrm{C}) \mathrm{w}$ el 4.45\%, semicálidos húmedos $(\mathrm{A}) \mathrm{C}(\mathrm{w})$ el $41.67 \%$, templados húmedos $\mathrm{Cw}$ el $18.33 \%$ y fríos $\mathrm{E}$, el $0.001 \%$. En orden descendente, el número de climas por región fue: Región Sur 21, Costa Sierra Occidental 19, Sierra de Amula 18, Norte 14, Sureste 13, Costa Sur 12, Altos Sur 11, Valles 10, Lagunas 9, Centro 8, Altos Norte 7 y Ciénega 7. Se concluye que el estado de Jalisco posee un gradiente térmico de cálido a frío, incluyendo la semicálida y templada y por un gradiente de humedad la semiárida hasta la subhúmeda monzónica. Los autores consideran que los mapas climáticos obtenidos, pueden ser útiles en aplicaciones para diversas áreas, como la agricultura, edafología, ecología y ciencias ambientales.

Palabras clave: clasificación climática, relación clima-vegetación, SIG.

Recibido: marzo de 2021

Aceptado: junio de 2021 


\section{Introducción}

El clima es uno de los componentes ambientales de mayor variación tanto en espacio como en tiempo, debido que es el resultado de la interacción de diversos factores como latitud, altitud, relieve y exposición del terreno, distribución de tierras y cuerpos de agua, vegetación, sistemas globales de distribución de corrientes marinas y vientos (Critchfield, 1983; García, 1989). Todos estos factores interactúan para formar múltiples combinaciones de condiciones ambientales que se reflejan en una amplia gama de variantes climáticas. Para entender la naturaleza y diferenciación de tales variantes, se recurre a los sistemas de clasificación climática (Griffiths, 1994).

La clasificación de los climas de una entidad geográfica es fundamental para caracterizar las condiciones ambientales prevalecientes de una manera sintética, pero a la vez suficientemente descriptiva. Existen diversos sistemas de clasificación climática, sin embargo, ninguno de ellos cubre todas las posibles necesidades de clasificación del clima (Medina et al., 1998). Dentro de los sistemas de clasificación de macroclimas, el sistema Köppen es el más utilizado mundialmente (Sánchez-Santillán y Garduño, 2008) ya que fue concebido para definir las grandes áreas climáticas de la Tierra (García, 2004), en función básicamente de una variación climática de origen latitudinal.

La amplitud del uso del sistema Köppen a escala mundial, se ha logrado no sin previas adaptaciones a las condiciones ambientales particulares de distintas regiones del mundo, para caracterizar satisfactoriamente la diversidad climática regional (Stern y de Hoedt, 2000). Este es el caso de México, en donde la variación espacial del clima obedece principalmente a factores orográficos, por lo que hubo necesidad de ser adecuaciones al sistema Köppen para representar de manera apropiada la diversidad climática del país. Esto se logró mediante los trabajos seriados y de mejora continua de García (2004). El resultado de este trabajo de décadas de García (2004) es un sistema de clasificación climática que difiere significativamente de la versión original de Köppen.

Mientras el sistema original está diseñado para definir la distribución planetaria de los climas a macroescala y básicamente en función de una variación latitudinal del clima, el sistema modificado por García permite representar con detalle, la distribución de climas en México a mesoescala, producto de considerar una variación espacial del clima en producto de la interacción de múltiples factores modificadores del clima como altitud, relieve, exposición del terreno, distancia al mar y vegetación. Por lo anterior, consideramos que se justifica ampliamente referirnos al sistema Köppen modificado por García como el sistema Köppen-García (2004). Con base en el sistema Köppen-García (2004) se elaboró y actualizo periódicamente la cartografía climática del país a las escalas 1:500 000 (CETENAP, 1970), 1:1 000000 (SPP, 1981) y 1:4 000000 (UNAM, 1991) y 1:1 000000 de nuevo (CONABIO, 1998).

El uso del sistema Köppen-García en México va más allá de la relación clima-vegetación, puesto que ha sido utilizado en aplicaciones agrícolas (Álvarez y Salazar, 2017) y que considera la orografía como factor climático determinante en el territorio nacional (Hernández-Cerda et al., 2018), también ha sido planteada su aplicación en la planeación urbana (Velázquez-Ruiz et al., 2012); asimismo, se tiene conocimiento de que el sistema Köppen-García hoy en día continua aplicándose en los campos de la geografía, geomorfología, ecología, biología, veterinaria y arquitectura (García, 2004). 
El objetivo de esta investigación fue actualizar el mapa de climas del estado de Jalisco de acuerdo con el sistema de clasificación climática Köppen-García. Para ello se planteó considerar una amplia base de datos climáticos con actualización a 2014, un sistema de información de alta resolución en ambiente de Sistemas de Información Geográfica (SIG) y el debido ajuste de los climas obtenidos, por su correspondencia con la vegetación potencial, según los acervos de la comisión técnico consultivo de coeficientes de agostadero (COTECOCA).

Para el estado de Jalisco se han desarrollado diversos estudios relacionados con la generación de cartografía climática y agroclimática (Villalpando y García, 1993; Ramírez-Sánchez et al., 2013); sin embargo, ninguno de ellos se ha enfocado específicamente en la actualización de la carta de climas de la entidad con base en el sistema Köppen-García.

\section{Materiales y métodos}

\section{Base de datos climáticos}

Se trabajó con datos diarios y mensuales de precipitación, temperatura máxima, temperatura mínima y evaporación de 305 estaciones meteorológicas distribuidas en el interior y en los alrededores del estado de Jalisco (Figura 1). La información correspondió al periodo 1961-2014 para las estaciones de Jalisco (154) y el periodo 1961-2010 para las estaciones de los estados vecinos (151). Todas las estaciones pertenecen a la red de monitoreo meteorológico del servicio meteorológico nacional. Para el caso del punto de máxima altitud en el estado, el cual es el pico sur del Nevado de Colima, no se contó con datos de una estación meteorológica por lo que se decidió utilizar información de la estación ubicada en el Nevado de Toluca, la cual está ubicada a una altitud de $4283 \mathrm{~m}$ muy similar a la del Nevado de Colima, que se ubica a $4330 \mathrm{msnm}$.

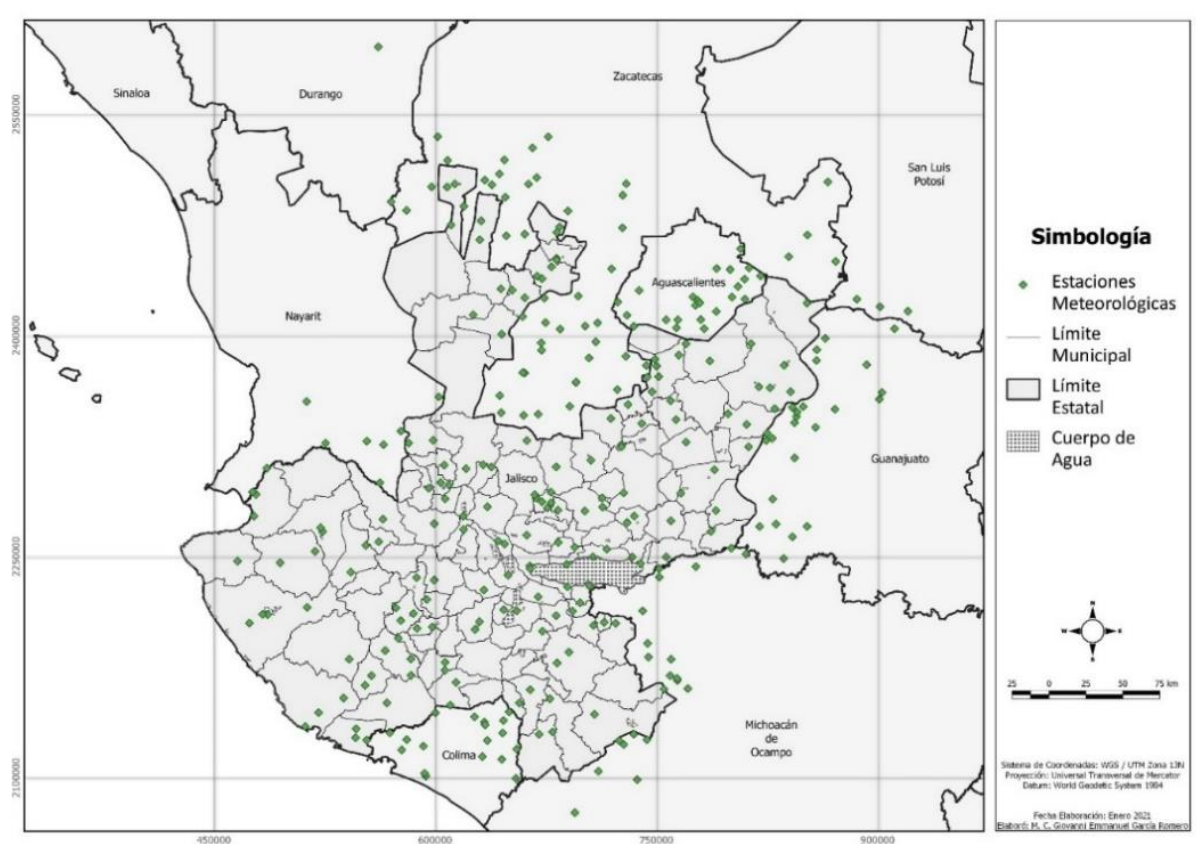

Figura 1. Distribución espacial de 305 estaciones meteorológicas utilizadas en el estudio. 


\section{Sistema de información climático}

A partir de los datos diarios se construyeron tablas dinámicas, para el cálculo de parámetros mensuales y anuales normales en Excel de Microsoft. Con las tablas normales mensuales de las 305 estaciones, se construyeron matrices de datos georreferenciados para proceder a continuación a implementar procesos de interpolación con base en el método Anusplin mediante el software Anusplin v. 4.4 (Hutchinson y Xu, 2020). Las interpolaciones se configuraron para generar imágenes con una resolución de $90 \mathrm{~m}$. Tanto para el caso de precipitación como de temperatura se trabajó con altitud como covariable.

Anusplin fue creado por Moradi Daleh y es un método ampliamente utilizado por diversas instituciones de prestigio como el Servicio Forestal de Canadá (Price et al., 2000), la Universidad Estatal de Oregón (Daly, 2006) y el Centro Nacional de Información Meteorológica de China (Xu et al., 2020). Anusplin puede modelar el efecto topográfico de manera efectiva involucrando el sistema coordenado tanto horizontal como vertical mientras realiza la interpolación de precipitación, además representa ventajas comparativas con relación a otros métodos de interpolación, debido a su simplicidad ya a que no requiere calibración de sus parámetros como en el caso del método Kriging. Mediante el método Anusplin se han generado varias de las rejillas (grids) más utilizadas de precipitación y temperatura en el mundo, este es el caso de WorldClim 1.0 (Hijmans et al., 2005) y WorldClim 2.0 (Fick y Hijmans, 2017).

Las salidas de los procesos de interpolación se recuperaron y documentaron como imágenes ráster en el sistema Idrisi Selva (Eastman, 2012), para integrar un sistema de información climático que originalmente se constituyó con 12 imágenes mensuales de temperatura máxima media, 12 de temperatura mínima media, 12 de precipitación acumulada promedio mensual y 12 de evaporación acumulada promedio mensual. A partir de las imágenes climáticas mensuales derivadas de las interpolaciones se calcularon en el sistema IDRISI Selva otras variables adicionales como temperatura media para cada mes y anual.

\section{Generación del mapa de climas de Jalisco}

A partir de las imágenes ráster mensuales y anuales de precipitación y temperatura media se procedió a la generación de un ráster de climas de Jalisco, mediante la aplicación del método de clasificación climática Köppen-García (García, 2004). Con fines de practicidad, se decidió representar en los resultados del procedimiento de clasificación climática, sólo los elementos de la fórmula climática correspondientes a los niveles de tipo y subtipo climático.

Para implementar el procedimiento de clasificación climática Köppen-García fue necesario generar además de las imágenes mensuales y anuales de temperatura media y precipitación, las imágenes de porcentaje de lluvia invernal, factor ' $r$ ', temperatura media del mes más cálido, temperatura media del mes más frío, precipitación del mes más seco, cociente precipitación anual/temperatura media anual (CPT) y número de meses con temperatura media $>10{ }^{\circ} \mathrm{C}$. Esto se realizó con el sistema Idrisi Selva mediante rutinas de álgebra de mapas utilizando los módulos overlay, scalar y reclass. Con la finalidad de automatizar el proceso espacial de clasificación climática y de facilitar la detección de errores en el proceso y su rápida corrección se elaboró un archivo macro (extensión. iml) a través del editor de archivos de Idrisi. 


\section{Análisis de correspondencia entre climas y vegetación potencial}

Una vez obtenido el ráster de climas de Jalisco, se analizó la correspondencia entre los climas obtenidos y la vegetación potencial según el mapa de tipos de vegetación de COTECOCA escala 1: 500 000. Para ello se sobrepusieron mediante álgebra de mapas el ráster de climas y el ráster de vegetación potencial, originando un nuevo ráster, del cual se extrajeron en formato Excel los climas y su correspondiente tipo de vegetación. Se corrigieron los climas que no correspondían con el tipo de vegetación propuesto por COTECOCA. En la memoria de coeficientes de agostadero del estado de Jalisco (COTECOCA, 1979), se incluye la descripción de las unidades geológicas, unidades de suelo y climas que se presentan en cada tipo de vegetación.

Además, se incluyen los sitios de pastizal con sus correspondientes coeficientes de agostadero. Cabe señalar, que fue necesario analizar cuantas variantes climáticas podían corresponder con algunos tipos de vegetación de cobertura territorial amplia, como es el caso de la selva baja caducifolia, para la cual se encontró que podía estar presente en diversos tipos climáticos (TrejoVázquez, 1999).

\section{Resultados y discusión}

De un total de 205 polígonos resultantes de la sobre-posición de la imagen de climas y la imagen de tipos de vegetación potencial, se obtuvo correspondencia en $84 \%$ de los casos (172 polígonos), esto es, que en 33 polígonos (10.7\%) hubo necesidad de reclasificar o ajustar el clima que se había obtenido sólo con el análisis de variables climáticas. De estos 33 polígonos, seis se refieren a un clima $\mathrm{BS}_{1}$ mal clasificado; seis al $\mathrm{Aw}, 14$ al clima $\mathrm{A}(\mathrm{C}) \mathrm{w}$ y siete casos al clima $(\mathrm{A}) \mathrm{C}(\mathrm{w})$.

De los 33 polígonos que fueron corregidos, ocho correspondieron a un cambio de clima semicálido del grupo C a semicálido del grupo A; seis polígonos pasaron de una clasificación de clima cálido A, a clima semicálido del grupo A; seis más correspondieron sólo a un cambio de subtipo del grupo semiárido $\mathrm{BS}_{1}$; cuatro casos implicaron un cambio de clima cálido $\mathrm{A}$, a semicálido del grupo $\mathrm{C}$; cuatro cambios más fueron de clima semiárido $\mathrm{BS}_{1}$ a clima cálido $\mathrm{A}$; tres polígonos de clima semicálido del grupo $\mathrm{C}$ sólo experimentaron un cambio a nivel de subtipo. También se presentó un cambio a nivel de subtipo de clima A y finalmente, ocurrió un sólo caso de cambio de clima templado $\mathrm{C}$ a clima semicálido del grupo A.

La Figura 2 muestra el mapa obtenido de climas de Jalisco; de acuerdo con este, existe en el Estado una gran diversidad climática, representada por un total de 29 variantes climáticas (Figura 2 y Cuadro 1), a nivel de tipo y subtipo climático, según el sistema Köppen-García (García, 2004). De esta manera, se puede apreciar que el clima (A)Ca( $\left.\mathrm{w}_{0}\right)$ es el de mayor cobertura superficial, ya que está presente en casi una quinta parte (18.35\%) del territorio jalisciense. Otros climas de importancia son el $(\mathrm{A}) \mathrm{Ca}\left(\mathrm{w}_{1}\right), \mathrm{Cb}\left(\mathrm{w}_{1}\right) \mathrm{Aw} \mathrm{w}_{1}$ y el $\mathrm{Aw}_{0}$ con una cobertura territorial de $12.57,9.62,9.41$ y $8.87 \%$ respectivamente. Estos cinco climas suman cerca de $60 \%$ de la superficie estatal. 


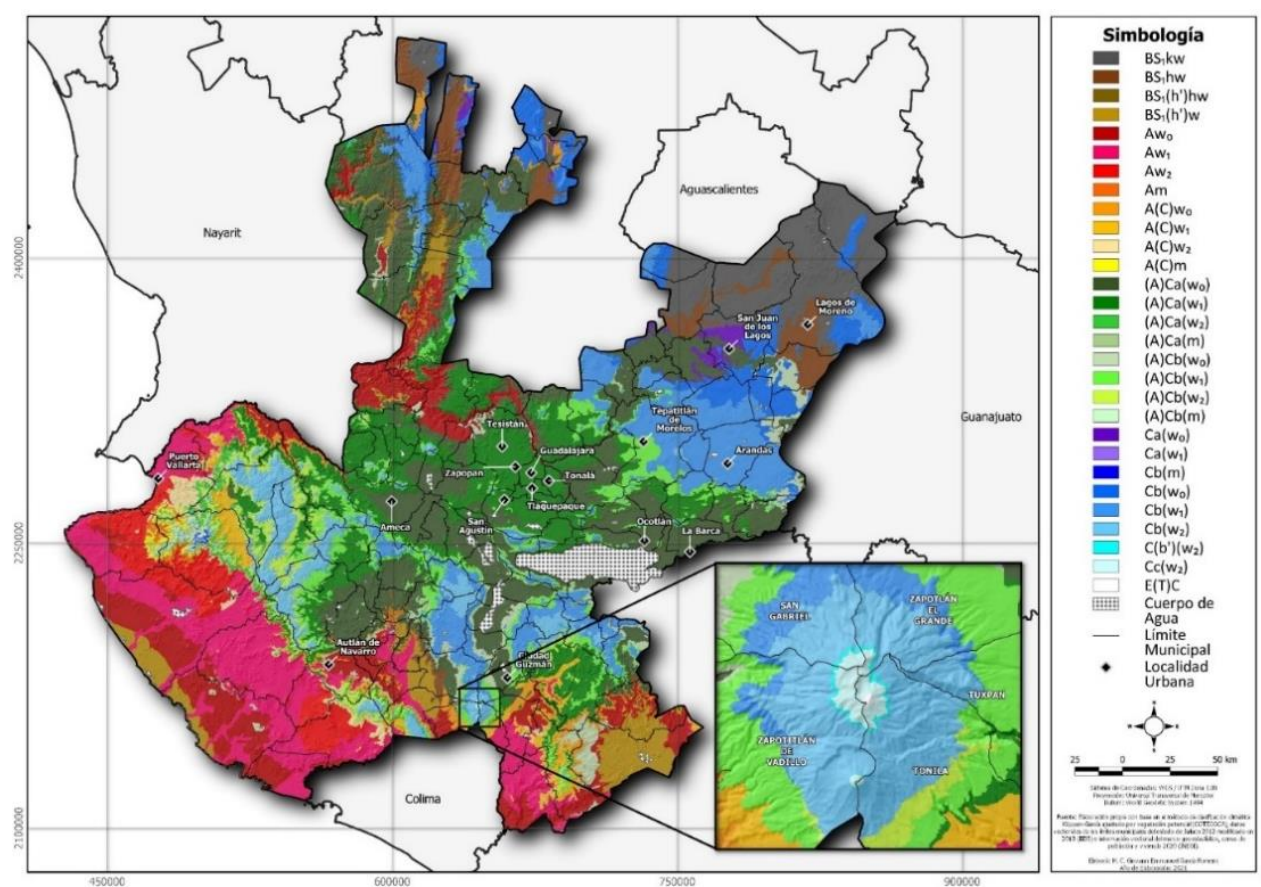

Figura 2. Climas de Jalisco según el sistema Köppen-García (2004).

Cuadro 1. Representatividad de los climas de Jalisco en términos de cobertura territorial.

\begin{tabular}{|c|c|c|c|c|c|}
\hline \multirow{2}{*}{ Clima } & \multicolumn{2}{|c|}{ Superficie estatal } & \multirow{2}{*}{ Clima } & \multicolumn{2}{|c|}{ Superficie estatal (\%) } \\
\hline & (ha) & $(\%)$ & & (ha) & $(\%)$ \\
\hline $\mathrm{BS}_{1} \mathrm{kw}$ & 543797.82 & 6.86 & $(\mathrm{~A}) \mathrm{Ca}(\mathrm{m})$ & 15835.33 & 0.2 \\
\hline $\mathrm{BS}_{1} \mathrm{hw}$ & 260948.56 & 3.29 & $(\mathrm{~A}) \mathrm{Cb}\left(\mathrm{w}_{0}\right)$ & 193105.16 & 2.44 \\
\hline $\mathrm{BS}_{1}\left(\mathrm{~h}^{\prime}\right) \mathrm{hw}$ & 16497.15 & 0.21 & $(\mathrm{~A}) \mathrm{Cb}\left(\mathrm{w}_{1}\right)$ & 415174.2 & 5.24 \\
\hline $\mathrm{BS}_{1}\left(\mathrm{~h}^{\prime}\right) \mathrm{w}$ & 270938.33 & 3.42 & $(\mathrm{~A}) \mathrm{Cb}\left(\mathrm{w}_{2}\right)$ & 156145.99 & 1.97 \\
\hline $\mathrm{Aw}_{0}$ & 702697.74 & 8.87 & $(\mathrm{~A}) \mathrm{Cb}(\mathrm{m})$ & 12214.29 & 0.15 \\
\hline $\mathrm{Aw}_{1}$ & 745402.32 & 9.41 & $\mathrm{Ca}\left(\mathrm{w}_{0}\right)$ & 52912.85 & 0.67 \\
\hline $\mathrm{Aw}_{2}$ & 258621.69 & 3.26 & $\mathrm{Ca}\left(\mathrm{w}_{1}\right)$ & 4.78 & 0.0001 \\
\hline Am & 18379.05 & 0.23 & $\mathrm{Cb}\left(\mathrm{w}_{0}\right)$ & 337244.46 & 4.26 \\
\hline $\mathrm{A}(\mathrm{C}) \mathrm{w}_{0}$ & 117981.09 & 1.49 & $\mathrm{Cb}\left(\mathrm{w}_{1}\right)$ & 762630.64 & 9.62 \\
\hline $\mathrm{A}(\mathrm{C}) \mathrm{w}_{1}$ & 139754.40 & 1.76 & $\mathrm{Cb}\left(\mathrm{w}_{2}\right)$ & 295057.66 & 3.72 \\
\hline $\mathrm{A}(\mathrm{C}) \mathrm{w}_{2}$ & 72729.63 & 0.92 & $\mathrm{Cb}(\mathrm{m})$ & 2220.39 & 0.03 \\
\hline $\mathrm{A}(\mathrm{C}) \mathrm{m}$ & 22000.78 & 0.28 & $\mathrm{C}\left(\mathrm{b}^{\prime}\right)\left(\mathrm{w}_{2}\right)$ & 472.70 & 0.01 \\
\hline$(\mathrm{A}) \mathrm{Ca}\left(\mathrm{w}_{0}\right)$ & 1453999.33 & 18.35 & $\mathrm{Cc}\left(\mathrm{w}_{2}\right)$ & 1361.76 & 0.02 \\
\hline$(\mathrm{A}) \mathrm{Ca}\left(\mathrm{w}_{1}\right)$ & 995844.42 & 12.57 & $\mathrm{E}(\mathrm{T}) \mathrm{C}$ & 59.59 & 0.001 \\
\hline$(\mathrm{A}) \mathrm{Ca}\left(\mathrm{w}_{2}\right)$ & 59656.04 & 0.75 & & & \\
\hline
\end{tabular}


Al analizar la representatividad de los climas en Jalisco, pero ahora por grupos de climas, se tiene que los climas secos o semiáridos representan $13.78 \%$ del territorio estatal, los climas cálido húmedo $\mathrm{A}$ el $21.77 \%$, los climas semicálidos húmedos $\mathrm{A}(\mathrm{C})$ el 4.45\%, los climas semicálidos húmedos (A)C 41.67\%, los climas templados húmedos C, $18.33 \%$ y los climas fríos $\mathrm{E}, 0.001 \%$.

Esto proporciona una clara idea sobre la climatología del estado y muestra la predominancia de los climas semicálidos en Jalisco, puesto que sumando los semicálidos del grupo C con los semicálidos del grupo A, resulta que aproximadamente la mitad (45.76\%) del territorio estatal posee un clima semicálido.

Esto resalta la importancia de las modificaciones que García (2004) realizó al sistema original de clasificación climática de Köppen, sin las cuales hoy sería difícil representar adecuadamente la diversidad climática del estado. La predominancia de climas semicálidos húmedos le provee a Jalisco una condición climática transicional entre climas cálidos y templados que resultan ideales para una gran diversidad de especies de plantas.

Los climas semicálidos, sobre todo los derivados del grupo $\mathrm{C}$, presentan días cálidos y noches frescas, lo que resulta en condiciones óptimas para especies termo-periódicas (Wendell et al., 2017) como es el caso del maíz (Benacchio, 1982), de ahí que el estado de Jalisco sea considerado como una de las regiones del país con mayor potencial de rendimiento para este cultivo (CastañedaZavala et al., 2014).

El Cuadro 2 y las Figuras de la 3 a la 8, muestran una visión de la diversidad climática a escala regional, además destacan a las regiones Sur, Costa Sierra Occidental y Sierra de Amula como las más diversas, debido a que albergan 21, 19 y 18 variantes climáticas respectivamente, esto es 72.4, 65.5 y $62.1 \%$ de la variación climática total del estado. De acuerdo con el Cuadro 2, la Región Altos Norte (R01) es predominantemente semiárida templada, ya que $56.36 \%$ de su territorio está ocupada por el clima $\mathrm{BS}_{1} \mathrm{kw}$, en tanto la Región Altos Sur (R02) es mayormente templada con verano fresco largo y un temporal de lluvias moderadamente húmedo, dado que $51.22 \%$ de su superficie está representada por el clima $\mathrm{Cb}\left(\mathrm{w}_{1}\right)$.

Para la Región Centro (R03) tanto como para la Región Valles (R12), el clima (A)Ca(w1) es el más representativo (58.39\% de la superficie regional), por lo que pueden referenciarse como regiones semicálidas, templadas con tendencia a cálidas y un temporal moderadamente húmedo. Las regiones Ciénega (R04), Lagunas (R07) y Norte (R08) pueden ser tipificadas también como regiones semicálidas, pero, con un temporal moderadamente seco, dada la predominancia del clima $(\mathrm{A}) \mathrm{Ca}\left(\mathrm{w}_{0}\right)$.

Las regiones Costa Sierra Occidental (R05) y Sur (R10) no presentan una clara predominancia de un clima, en parte debido a su alta diversidad ambiental (19 y 21 climas, respectivamente), pero, por otro lado, esta característica las convierte en regiones que pueden albergar una diversidad florística importante (Suárez-Mota et al., 2017). Más de 72\% de la superficie de la Región Costa Sur (R06) presenta un clima cálido Aw (Aw $147.61 \%$ y Aw $25.05 \%$ de la superficie). Por último, en la Región Sierra de Amula (R09) predominan tres variantes del clima (A)C, en tanto en la Región Sureste (R11) predominan cuatro climas que cubren una gama que va del Aw hasta el templado $\mathrm{Cb}(\mathrm{w})$. 
Cuadro 2. Porcentaje territorial de diversos climas en las 12 regiones ecológicas de Jalisco.

\begin{tabular}{|c|c|c|c|c|c|c|c|c|c|c|c|c|}
\hline \multirow{2}{*}{ Clima } & \multicolumn{12}{|c|}{ Regiones } \\
\hline & R01 & R02 & R03 & R04 & R05 & R06 & R07 & R08 & R09 & R10 & R11 & $\mathrm{R} 12$ \\
\hline $\mathrm{BS}_{1} \mathrm{kw}$ & 56.36 & 0.01 & & & & & & 5.97 & 0.02 & 0.04 & & \\
\hline $\mathrm{BS}_{1} \mathrm{hw}$ & 13.51 & 0.06 & & & & & 0.12 & 12.46 & 0.74 & 1.28 & & \\
\hline $\mathrm{BS}_{1}\left(\mathrm{~h}^{\prime}\right) \mathrm{hw}$ & & & & & & & 0.02 & 1.59 & & & & \\
\hline $\mathrm{BS}_{1}\left(\mathrm{~h}^{\prime}\right) \mathrm{w}$ & & & & & & 6.44 & & 4.41 & 4.08 & 13.41 & 8.12 & \\
\hline $\mathrm{Aw}_{0}$ & & & 3.71 & & 4.56 & 25.05 & & 6.84 & 9.69 & 13.17 & 12.23 & 22.8 \\
\hline $\mathrm{Aw}_{1}$ & & & & & 14.39 & 47.61 & & & 6.66 & 13.7 & 0.002 & \\
\hline $\mathrm{Aw}_{2}$ & & & & & 15.24 & 12.38 & & & 0.04 & 0.01 & & \\
\hline $\mathrm{Am}$ & & & & & 2.03 & & & & & & & \\
\hline $\mathrm{A}(\mathrm{C}) \mathrm{w}_{0}$ & & & & & 1.93 & & & 0.11 & 2.41 & 5.49 & 3.67 & 1.26 \\
\hline $\mathrm{A}(\mathrm{C}) \mathrm{w}_{1}$ & & & & & 5.35 & 0.92 & & & 1.71 & 8.53 & 0.11 & 0.01 \\
\hline $\mathrm{A}(\mathrm{C}) \mathrm{w}_{2}$ & & & & & 4.52 & 2.48 & & & 0.42 & 0.62 & & \\
\hline $\mathrm{A}(\mathrm{C}) \mathrm{m}$ & & & & & 2.42 & & & & & & & \\
\hline$(\mathrm{A}) \mathrm{Ca}\left(\mathrm{w}_{0}\right)$ & 4.1 & 22.25 & 23.30 & 62.24 & 0.48 & & 48.96 & 34.22 & 28.07 & 5.63 & 19.52 & 24.9 \\
\hline$(\mathrm{A}) \mathrm{Ca}\left(\mathrm{w}_{1}\right)$ & & 2.87 & 58.39 & 19.2 & 11.01 & 0.48 & 7.71 & 3.86 & 13.77 & 11.97 & 4.99 & 44.3 \\
\hline$(\mathrm{A}) \mathrm{Ca}\left(\mathrm{w}_{2}\right)$ & & & & & 4.81 & 1.21 & & & 0.65 & 0.06 & & \\
\hline$(\mathrm{A}) \mathrm{Ca}(\mathrm{m})$ & & & & & 1.75 & & & & & & & \\
\hline$(\mathrm{A}) \mathrm{Cb}\left(\mathrm{w}_{0}\right)$ & 3.12 & 2.65 & 1.3 & 1.2 & 0.002 & 0.6 & 6.46 & 1.35 & 2.57 & 7.13 & 4.83 & 0.78 \\
\hline$(\mathrm{A}) \mathrm{Cb}\left(\mathrm{w}_{1}\right)$ & & 7.69 & 8.26 & 10.49 & 4.08 & 0.005 & 8.45 & 2.62 & 11.83 & 7.77 & 12 & 3.71 \\
\hline$(\mathrm{A}) \mathrm{Cb}\left(\mathrm{w}_{2}\right)$ & & & 0.0003 & & 11.13 & 1.75 & & & 3.63 & 1.56 & 0.68 & 0.33 \\
\hline$(\mathrm{A}) \mathrm{Cb}(\mathrm{m})$ & & & & & 1.35 & & & & & & & \\
\hline $\mathrm{Ca}\left(\mathrm{w}_{0}\right)$ & 2.93 & 2.18 & & & & & & 3.32 & & & & \\
\hline $\mathrm{Ca}\left(\mathrm{w}_{1}\right)$ & & 0.0007 & & & & & & & & & & \\
\hline $\mathrm{Cb}\left(\mathrm{w}_{0}\right)$ & 16.78 & 10.69 & & 0.24 & & & 0.17 & 11.5 & 0.004 & 0.05 & 0.58 & \\
\hline $\mathrm{Cb}\left(\mathrm{w}_{1}\right)$ & 3.2 & 51.22 & 4.76 & 6.63 & 0.28 & & 21.41 & 11.75 & 3.75 & 5.59 & 24.05 & 0.65 \\
\hline $\mathrm{Cb}\left(\mathrm{w}_{2}\right)$ & & 0.37 & 0.28 & 0.003 & 14.42 & 1.08 & 6.7 & 0.001 & 9.95 & 3.76 & 9.22 & 1.25 \\
\hline $\mathrm{Cb}(\mathrm{m})$ & & & & & 0.24 & & & & & & & \\
\hline $\mathrm{C}\left(\mathrm{b}^{\prime}\right)\left(\mathrm{w}_{2}\right)$ & & & & & & & & & & 0.06 & & \\
\hline $\mathrm{Cc}\left(\mathrm{w}_{2}\right)$ & & & & & & & & & & 0.16 & & \\
\hline $\mathrm{E}(\mathrm{T}) \mathrm{C}$ & & & & & & & & & & 0.01 & & \\
\hline Total & 7 & 11 & 8 & 7 & 19 & 12 & 9 & 14 & 18 & 21 & 13 & 10 \\
\hline
\end{tabular}



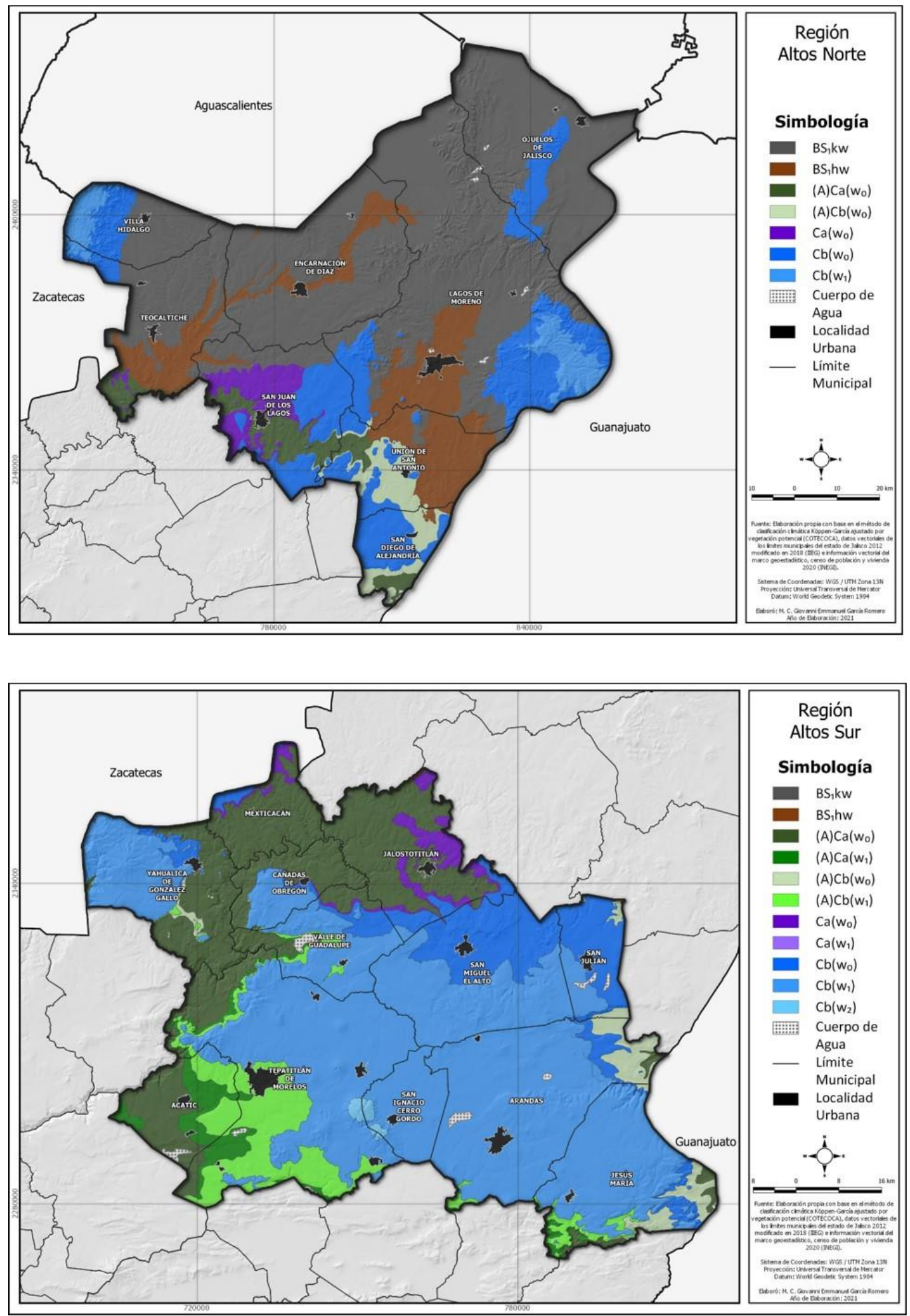

Figura 3. Diversidad climática de las Regiones Altos Norte y Altos Sur. 

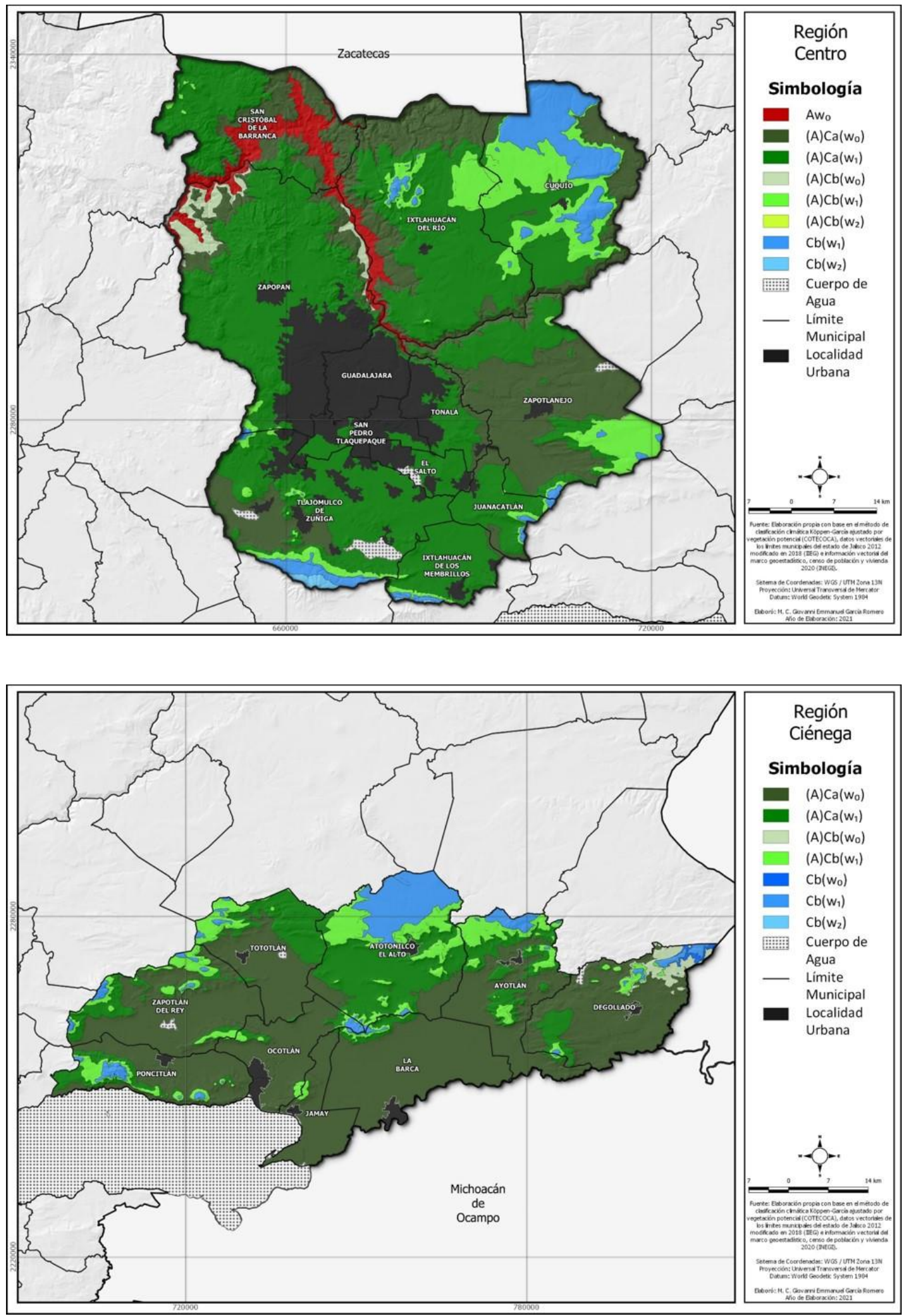

Figura 4. Diversidad climática de las Regiones Centro y Ciénega. 

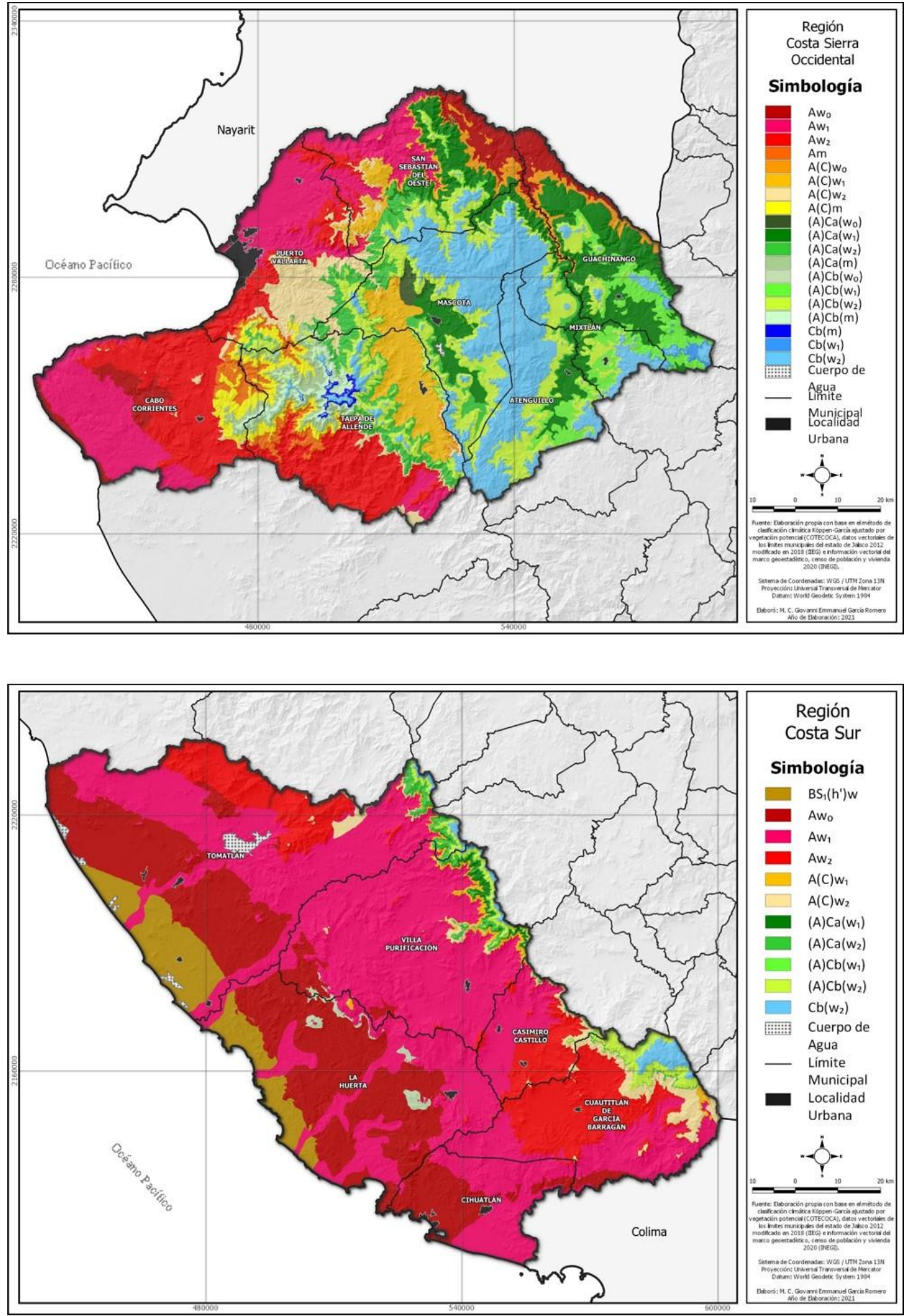

Figura 5. Diversidad climática de las Regiones Costa Sierra Occidental y Costa Sur. 

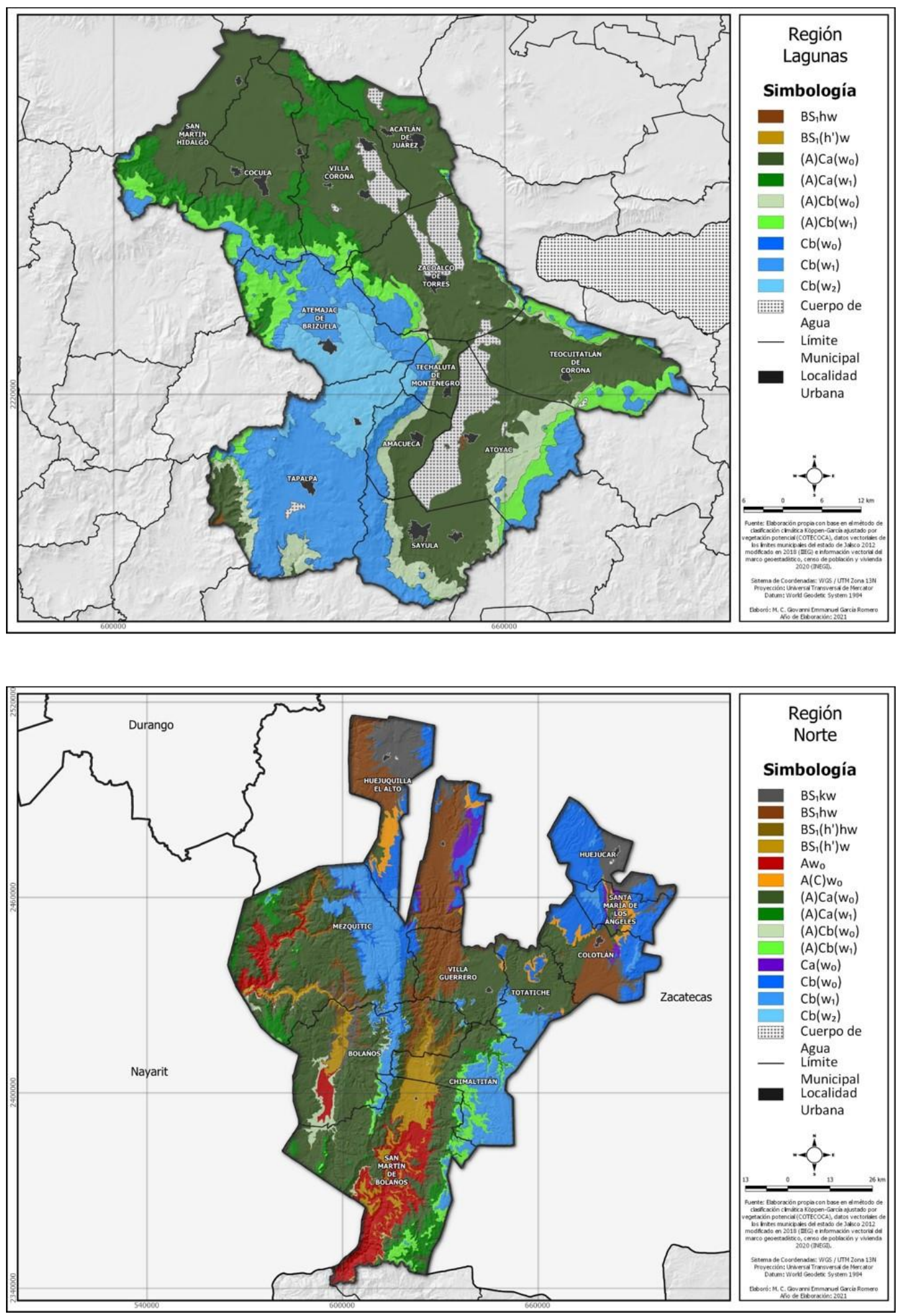

Figura 6. Diversidad climática de las Regiones Lagunas y Norte. 

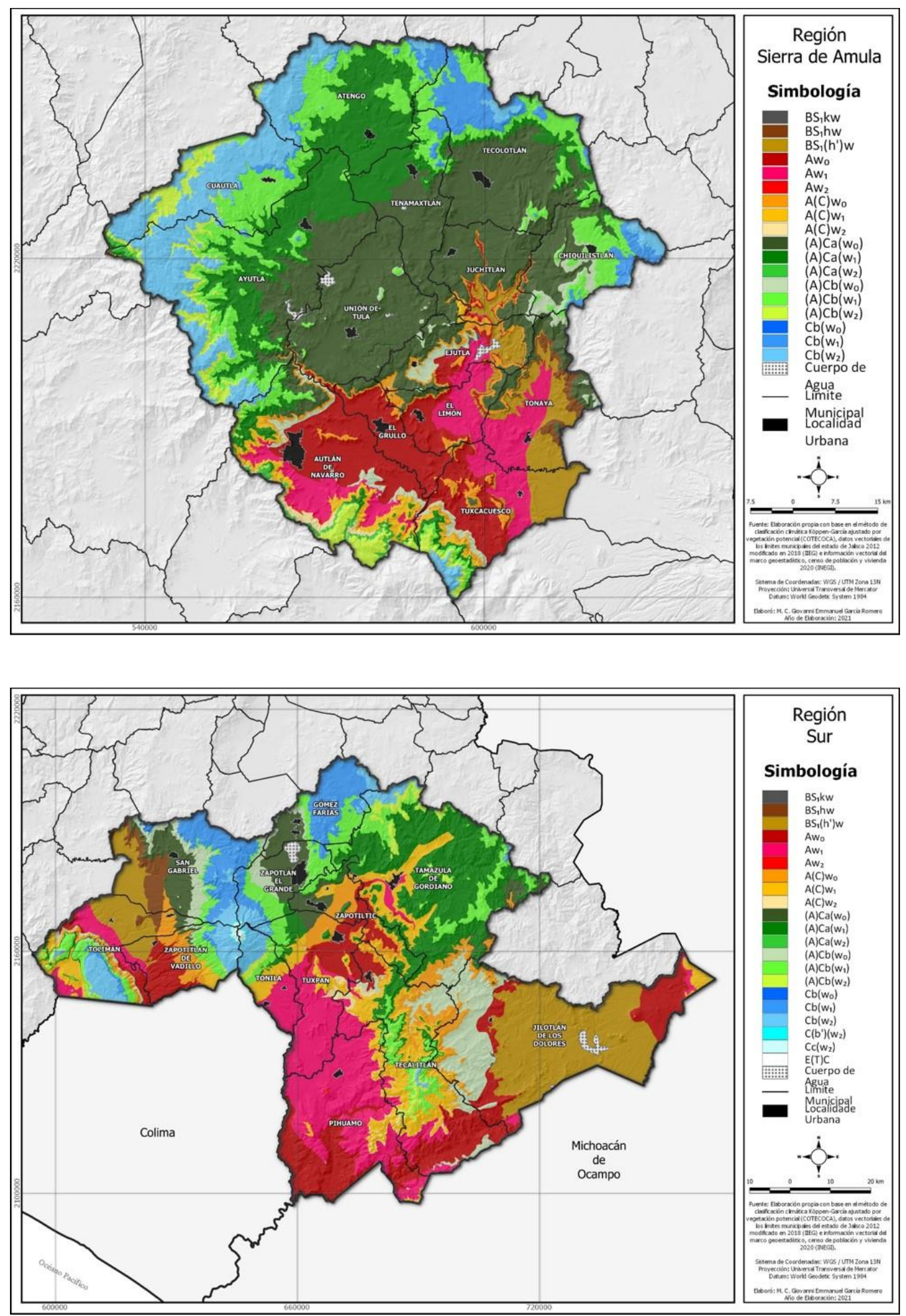

Figura 7. Diversidad climática de las Regiones Sierra de Amula y Sur. 

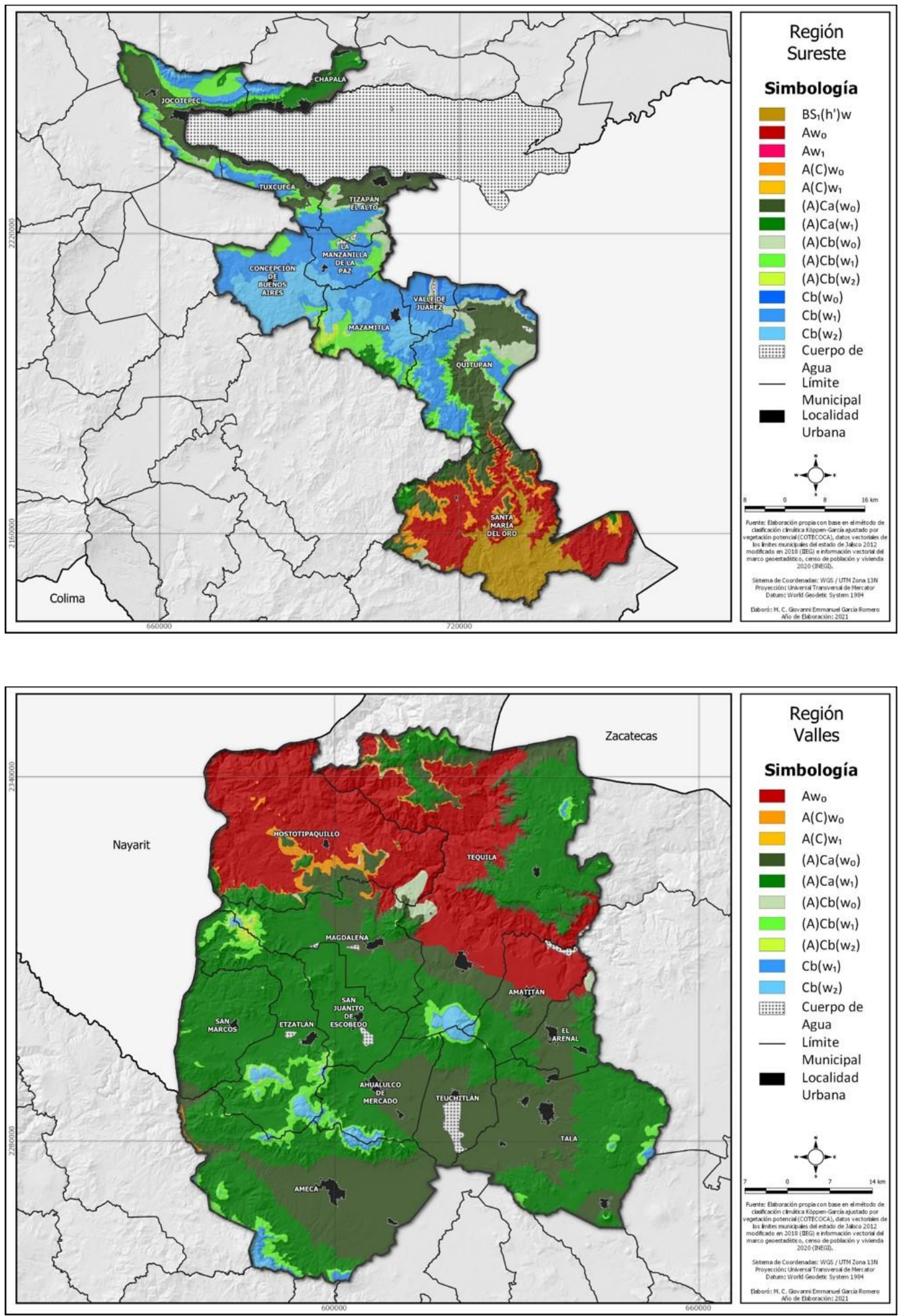

Figura 8. Diversidad climática de las Regiones Sureste y Valles. 
La descripción de los 29 climas obtenidos en este estudio se provee en el Cuadro 3. Nota: Los mapas generados pueden solicitarse a: ariel.ruiz@academicos.udg.mx y sergio.contreras@academicos.udg.mx.

Cuadro 3. Descripción de 29 climas del estado de Jalisco.

\begin{tabular}{|c|c|}
\hline Clima & Descripción \\
\hline $\mathrm{BS}_{1} \mathrm{kw}$ & $\begin{array}{l}\text { Semiárido templado con verano cálido (TMA } 12-18{ }^{\circ} \mathrm{C} \text {; TMMC } \geq 18{ }^{\circ} \mathrm{C} \text { ); lluvias de } \\
\text { verano }\end{array}$ \\
\hline $\mathrm{BS}_{1} \mathrm{hw}$ & Semiárido semicálido (TMA $18-22^{\circ} \mathrm{C}$; TMMF $<18^{\circ} \mathrm{C}$ ), lluvias de verano \\
\hline $\mathrm{BS}_{1}\left(\mathrm{~h}^{\prime}\right) \mathrm{hw}$ & Semiárido cálido $\left(\mathrm{TMA} \geq 22^{\circ} \mathrm{C} ; \mathrm{TMMF}<18^{\circ} \mathrm{C}\right)$, lluvias de verano \\
\hline $\mathrm{BS}_{1}\left(\mathrm{~h}^{\prime}\right) \mathrm{w}$ & Semiárido cálido $\left(\mathrm{TMA} \geq 22^{\circ} \mathrm{C} ; \mathrm{TMMF} \geq 18^{\circ} \mathrm{C}\right)$, lluvias de verano \\
\hline $\mathrm{Aw}_{0}$ & Cálido con lluvias de verano; CPT $<43.2$ \\
\hline $\mathrm{Aw}_{1}$ & Cálido con lluvias de verano; CPT entre 43.2 y 55.3 \\
\hline $\mathrm{Aw}_{2}$ & Cálido con lluvias de verano; CPT> 55.3 \\
\hline Am & Cálido con lluvias monzónicas de verano \\
\hline $\mathrm{A}(\mathrm{C}) \mathrm{w}_{0}$ & Semicálido del grupo A con lluvias de verano; CPT $<43.2$ \\
\hline $\mathrm{A}(\mathrm{C}) \mathrm{w}_{1}$ & Semicálido del grupo A con lluvias de verano; CPT entre 43.2 y 55.3 \\
\hline $\mathrm{A}(\mathrm{C}) \mathrm{w}_{2}$ & Semicálido del grupo A con lluvias de verano; CPT> 55.3 \\
\hline $\mathrm{A}(\mathrm{C}) \mathrm{m}$ & Semicálido del grupo A con lluvias monzónicas de verano \\
\hline$(\mathrm{A}) \mathrm{Ca}\left(\mathrm{w}_{0}\right)$ & Semicálido con verano cálido; lluvias de verano; CPT $<43.2$, TMMC $>22^{\circ} \mathrm{C}$. \\
\hline$(\mathrm{A}) \mathrm{Ca}\left(\mathrm{w}_{1}\right)$ & Semicálido con verano cálido; lluvias de verano; CPT de 43.2 a 55, TMMC $>22^{\circ} \mathrm{C}$ \\
\hline$(\mathrm{A}) \mathrm{Ca}\left(\mathrm{w}_{2}\right)$ & Semicálido con verano cálido; lluvias de verano; CPT $>55$, TMMC $>22{ }^{\circ} \mathrm{C}$ \\
\hline$(\mathrm{A}) \mathrm{Ca}(\mathrm{m})$ & Semicálido con verano cálido; lluvias monzónicas de verano y $\mathrm{TMMC}>22{ }^{\circ} \mathrm{C}$ \\
\hline$(\mathrm{A}) \mathrm{Cb}\left(\mathrm{w}_{0}\right)$ & Semicálido con verano fresco largo; lluvias de verano; $\mathrm{CPT}<43.2, \mathrm{TMMC}<22{ }^{\circ} \mathrm{C}$. \\
\hline$(\mathrm{A}) \mathrm{Cb}\left(\mathrm{w}_{1}\right)$ & $\begin{array}{l}\text { Semicálido con verano fresco largo; lluvias de verano; CPT de } 43.2 \text { a 55, TMMC }< \\
22{ }^{\circ} \mathrm{C}\end{array}$ \\
\hline$(\mathrm{A}) \mathrm{Cb}\left(\mathrm{w}_{2}\right)$ & Semicálido con verano fresco largo; lluvias de verano; CPT $>55$, TMMC $<22{ }^{\circ} \mathrm{C}$ \\
\hline$(\mathrm{A}) \mathrm{Cb}(\mathrm{m})$ & Semicálido con verano fresco largo; lluvias monzónicas de verano; TMMC $<22{ }^{\circ} \mathrm{C}$ \\
\hline $\mathrm{Ca}\left(\mathrm{w}_{0}\right)$ & Templado con verano cálido; lluvias de verano; TMMC $>22^{\circ} \mathrm{C}, \mathrm{CPT}<43.2$ \\
\hline $\mathrm{Ca}\left(\mathrm{w}_{1}\right)$ & Templado con verano cálido; lluvias de verano; TMMC> $22{ }^{\circ} \mathrm{C}$, CPT de 43.2 a 55 \\
\hline $\mathrm{Cb}\left(\mathrm{w}_{0}\right)$ & Templado con verano fresco largo; lluvias de verano; TMMC $<22{ }^{\circ} \mathrm{C}, \mathrm{CPT}<43.2$ \\
\hline $\mathrm{Cb}\left(\mathrm{w}_{1}\right)$ & $\begin{array}{l}\text { Templado con verano fresco largo; lluvias de verano; TMMC }<22{ }^{\circ} \mathrm{C} \text {, CPT de } 43.2 \\
\text { a } 55\end{array}$ \\
\hline $\mathrm{Cb}\left(\mathrm{w}_{2}\right)$ & Templado con verano fresco largo; lluvias de verano; TMMC $<22{ }^{\circ} \mathrm{C}, \mathrm{CPT}>55$ \\
\hline $\mathrm{Cb}(\mathrm{m})$ & Templado con verano fresco largo; lluvias monzónicas de verano; TMMC $<22{ }^{\circ} \mathrm{C}$ \\
\hline $\mathrm{C}\left(\mathrm{b}^{\prime}\right)\left(\mathrm{w}_{2}\right)$ & Semifrío con verano fresco largo. TMA de 5 a $12^{\circ} \mathrm{C},>4$ meses $>10^{\circ} \mathrm{C}$ \\
\hline $\mathrm{Cc}\left(\mathrm{w}_{2}\right)$ & Semifrío con verano fresco corto. TMA de 5 a $12^{\circ} \mathrm{C},<4$ meses $>10^{\circ} \mathrm{C}$ \\
\hline $\mathrm{E}(\mathrm{T}) \mathrm{C}$ & Clima frío, con TMA de -2 a $5{ }^{\circ} \mathrm{C}$ y el $\mathrm{MMF}>0{ }^{\circ} \mathrm{C}$ \\
\hline
\end{tabular}

$\mathrm{TMA}=$ temperatura media anual; $\mathrm{TMMF}=$ temperatura media del mes más frío; $\mathrm{TMMC}=$ temperatura media del mes más cálido; $\mathrm{CPT}=$ cociente precipitación anual/temperatura media anual. 


\section{Conclusiones}

Se actualizó el mapa de climas de Jalisco ajustado a la vegetación potencial. El mapa de tipos de clima obtenido evidencia la diversidad climática del estado, representada por 29 variantes, que incluyen una gama térmica que va desde los climas cálidos hasta los climas fríos, incluyendo los semicálidos y los templados.

En cuanto a humedad, se tienen desde climas semiáridos hasta tipo monzónicos, abarcando los subhúmedos con un gradiente de humedad disponible <43.2 a >55 CPT. Cerca de $14 \%$ de la superficie estatal son áreas semiáridas y el resto del territorio es de clima húmedo. Los climas semicálidos subhúmedos son los más representativos, ya que cubren casi la mitad de la superficie estatal.

Las regiones con mayor diversidad climática son la Región Sur, la Región Costa Sierra Occidental y la Región Sierra de Amula con 21, 19 y 18 variantes climáticas, de las 29 presentes en el estado. Mientras que las regiones con menor diversidad climática son la Región Altos Norte, Región Centro y Región Ciénega con 7, 8 y 7 variantes climáticas, respectivamente.

Los mapas climáticos obtenidos, tanto el estatal como los regionales, presentan aplicaciones potenciales en diversas áreas, tales como la agrícola, para la posible definición de áreas potenciales de cultivos o la edafológica, para la generación de un mapa de zonas agroecológicas.

\section{Literatura citada}

Álvarez, B. A. y Salazar-García, S. 2017. Las condiciones ambientales determinan la rugosidad de la piel del fruto de aguacate 'Hass'. Rev. Mex. Cienc. Agric. 19(Esp):4063-4073.

Benacchio, S. S. 1982. Algunas exigencias agroecológicas en 58 especies de cultivo con potencial de producción en el Trópico Americano. FONAIAP-Centro Nal. de Inv. Agropecuarias. Ministerio de Agricultura y Cría. Maracay, Venezuela. 202 p.

Castañeda-Zavala, Y.; González-Merino, A.; Chauvet-Sánchez, M. y Ávila-Castañeda, J. F. 2014. Industria semillera de maíz en Jalisco. Actores sociales en conflicto. Sociológica. 29(83):241-278.

CETENAP. 1970. Comisión de Estudios del Territorio Nacional y Planeación (CETENAP). 1970. Cartas de climas, escala 1:500 000. México, DF.

CONABIO. Comisión Nacional para la Biodiversidad. 1998. Carta de Climas de la República Mexicana, Escala 1:1’000,000, según el Sistema de E. García. Comisión Nacional para la Biodiversidad (CONABIO). México, DF.

COTECOCA. 1979. Comisión Técnica Consultiva de Coeficientes de Agostadero. Mapa de tipos de vegetación escala 1:500,000. En: Memoria de Coeficientes de Agostadero del Estado de Jalico, Tomo I y Tomo II. Comisión Técnica Consultiva de Coeficientes de Agostadero (COTECOCA). Secretaría de Agricultura y Recursos Hidráulicos. México, DF.

Critchfield, H. J. 1983. General climatology. $4^{\text {th }}$ Ed. Prentice Hall. New Jersey, USA. 453 p.

Eastman, J. R. 2012. Idrisi Selva Manual. Clark University. Worcester, MA, USA. 322 p.

Fick, S. E. and Hijmans, R. J. 2017. WorldClim 2: new 1-km spatial resolution climate surfaces for global land areas. Int. J. Climatol. 37(12):4302-4315. Doi: 10.1002/joc.5086.

García, E. 1989. Apuntes de climatología. 6ª (Ed.). Offset Larios. México, DF. 155 p. 
García, E. 2004. Carta de climas Jalisco. Climatología de la República Mexicana. Proyecto CONABIO-Estadigrafía. México, DF. 90 p.

Griffiths, J. F. 1994. Climate classifications. In: handbook of agricultural meteorology. John F. Griffiths. Ed. Oxford Univ. Press. New York, USA. 133-147 pp.

Hernández-Cerda, M. E.; Ordoñez-Díaz, M. J. y Giménez de Azcárate, J. 2018. Análisis comparativo de dos sistemas de clasificación bioclimática aplicados en México. Investigaciones Geográficas. 95:1-14. Doi: 10.14350/rig.57451.

Hijmans, R. J.; Cameron, S. E.; Parra, J. L.; Jones, P. G. and Jarvis, A. 2005. Very high resolution interpolated climate surfaces for global land areas. Int. J. Climatol. 25(15):1965-1978.

Hutchinson, M. F. and Xu, T. 2013. Anusplin Version 4.4. User Guide. The Australian National University. Fenner School of Environment and Society. Canberra, Australia. 52 p.

Medina, G. G.; Ruiz, C. J. A. y Martínez, P. R. A. 1998. Los climas de México: una estratificación ambiental basada en el componente climático. Guadalajara, Jalisco, México. INIFAPCIRPAC. Guadalajara, Jalisco, México. 103 p.

Price, D. T.; McKenney, D.W.; Nalder, I. A.; Hutchinson, M. F. and Kesteven, J. L. 2000. A comparison of two statistical methods for spatial interpolation of Canadian monthly mean climate data. Agric. For. Meteorol. 101(2-3):81-94.

Ramírez-Sánchez, H. U.; Meulenert-Peña, A. R. y Gómez-Reyna, J. A. 2013. Actualización del atlas bioclimático del estado de Jalisco. Investigaciones Geográficas. Boletín. 82:66-92.

Sánchez-Santillán, N. y Garduño, L. R. 2008. Algunas consideraciones acerca de los sistemas de clasificación climática. ContactoS. 68:5-10.

SPP. 1981. Secretaría de Programación y Presupuesto. Síntesis geográfica del Estado de Jalisco (anexo cartográfico escala 1:1'000,000). Secretaría de Programación y Presupuesto (SPP) Coordinación General de los Servicios Nacional de Estadística, Geografía e Informática. México, DF. 22 p.

Stern, H. and de Hoedt, G. 2000. Objective classification of Australian climates. Aust. Met. Mag. 49(2):87-96.

Suárez-Mota, M. E.; Villaseñor, J. L. y López-Mata, L. 2017. Dominios climáticos de la Sierra Madre Oriental y su relación con la diversidad florística. Rev. Mex. Biod. 88(1):224-233. doi.org/10.1016/j.rmb.2017.01.020.

Trejo-Vázquez, I. 1999. El clima de la selva baja caducifolia en México. Investigaciones Geográficas, Boletín. 39:40-52.

UNAM. 1991. Universidad Nacional Autónoma de México. Atlas Nacional de México. Instituto de Geografía- Universidad Nacional Autónoma de México. https://geodigital.igg.unam.mx /atlas_nacional/index.html/grals/Tomo_II/IV.Naturaleza/IV.4.Clima/IV.4.10.jpg.

Velázquez-Ruiz, A.; Martínez, R. L. M. y Carrillo-González, F. M. 2012. Caracterización climática para la región de Bahía de Banderas mediante el sistema de Köppen, modificado por García, y técnicas de sistemas de información geográfica. Investigaciones Geográficas. 79:7-19.

Villalpando, I. J. F. y García, E. 1993. Agroclimatología del Estado de Jalisco. Universidad de Guadalajara, Centro universitario de Ciencias Biológicas y Agropecuarias. Guadalajara, Jalisco, México. 40 p.

Wendell, M.; Ripel, L.; Lee, Y. K.; Rognli, O. A.; Torre, S. and Olsen, J. E. 2017. Thermoperiodic control of floral induction involves modulation of the diurnal flowering locus $\mathrm{T}$ expression pattern. Plant Cell Physiol. 58(3):466-477. Doi: 10.1093/pcp/pcw221.

Xu, Y.; Zhao, P.; Si, D.; Cao, L.; Wu, X.; Zhao, Y. and Liu, N. 2020. Development and preliminary application of a gridded Surface air temperature homogenized dataset for China. Theor. Appl. Climatol. 139(1-2):505-516. https://doi.org/10.1007/s00704-019-02972-z. 